\title{
DESARROLLO APLICATIVO MÓVIL PARA LA GESTIÓN DE PEDIDOS EN LAS PYMES-CASO DE ESTUDIO FÁBRICA DE PINTURA INDUBER
}

Diana Marcela Vásquez Pérez, Michel Chilito Sandoval, Tania Isadora Mora Pedreros.

Semillero ITmedia

Grupo de Investigación Grintic

Institución Universitaria Antonio José Camacho

Recepción: 29/07/2019. Aceptado: 08/10/2019.

Cómo citar este artículo:

Vásquez Pérez, D.M., Chilito Sandoval, M. y Mora Pedreros, T.I. (2019). Desarrollo aplicativo móvil para la gestión de pedidos en las pymes-caso de estudio fábrica de pintura Induber. Revista Sapientía, 11 (22), 28-37.

\section{RESUMEN}

El presente artículo plantea una solución a la automatización de procesos para las PYMES que actualmente se encuentran en una etapa o fase de apertura, aquellas que nacen en muchos casos de ideas familiares que desean entrar en emprendimiento y lograr así su proyección en el mercado. Tomando como base este contexto, existen muchas microempresas que, debido a su falta de experiencia, manejo y gestión de la información, realizan sus procesos de manera convencional, de ahí que sus procesos sean manuales y consignados en papel en un gran porcentaje.

El desarrollo de esta aplicación se enfoca principalmente a la automatización del proceso de gestión de pedidos, parte fundamental en toda PYMES. Para resolver la problemática existente se optó por el desarrollo de un aplicativo móvil empleando nuevas tecnologías con fácil acceso a clientes, empleados y administradores, permitiendo con esto que dichas empresas logren una mayor proyección y puedan entrar en el mercado de una manera más agradable para sus clientes.

Para el desarrollo de la aplicación se utilizó la metodología ICONIX, la cual realiza los avances del proyecto mediante fases que se componen de unos artefactos específicos para lograr un desarrollo adecuado, rápido y eficiente.

\section{PALABRAS CLAVES}

PYMES, ICONIX, nuevas tecnologías, aplicación móvil.

\section{ABSTRACT}

The present project proposes a solution to the automation of processes for PYMES that are currently in an opening phase or stage, those that are born in many cases of family ideas that want to enter into entrepreneurship and thus achieve their projection in the market. Based on this context, there are many micro-enterprises which due to their lack of experience and information management carry out their processes in a conventional manner, hence their processes are manual and recorded on paper in a large percentage. 
The development of this application, it is focusing mainly on the automation of the order management process, a fundamental part of all PYMES. To solve the existing problem, it was decided to develop a mobile application using new technologies with easy access to customers, employees and administrators, thereby allowing these companies to achieve greater projection and be able to enter the market in a more pleasant way for their customers.

For the development of the application, the ICONIX methodology was used, which makes the progress of the project through phases that are made up of specific artifacts to achieve an adequate, fast and efficient development.

\section{KEYWORDS}

PYMES, ICONIX, new technologies, mobile application.

\section{INTRODUCCIÓN}

Según un análisis realizado por la Cámara de Comercio de Cali, las microempresas constituyen el mayor número de empresas registradas en Cali, empresas que permiten la generación de empleo y un crecimiento económico para la región (Cámara de Comercio Cali, 2018); muchas de estas microempresas debido a su desconocimiento realizan sus procesos de forma manual, lo que ocasiona la pérdida de información que podría ser de gran valor para los administradores, dueños y empleados. En este caso particular, se establece como punto de partida los pedidos que, en algún momento, luego de realizados por el cliente y entregados se pierde toda su información: productos que no se han ingresado al inventario, fechas de entregas y facturación. En cualquier caso, la perdida de la información mencionada anteriormente puede suponer un problema para la empresa o administrador debido a que en el caso extremo puede perder clientes, credibilidad y respeto hacia la empresa.
Al problema anterior se puede sumar un nuevo factor, las nuevas tecnologías, este factor es de gran importancia debido a que en esta época las microempresas se ven en la necesidad de automatizar los procesos y actividades que se llevan a cabo dentro de ellas. Este artículo se centrará en dar a conocer el proceso y la metodología para el desarrollo de la aplicación móvil centrada en el proceso de toma de pedidos, teniendo en cuenta las causas y consecuencias de realizar este proceso en forma manual, de acuerdo con la información suministrada por el dueño de la fábrica de pintura INDUBER ubicada en Cali.

Como un aporte para la solución de esta problemática se desarrollará un software bajo la metodología ICONIX, la cual permite mediante casos de uso tener un desarrollo más rápido y flexible a posibles modificaciones en el futuro. Este software permitirá a las PYMES sistematizar todo el proceso de toma de pedidos.

Sistematizar el registro de la toma de pedidos reducirá significativamente los problemas e inconvenientes que posee la empresa, dado que al tener procesos más óptimos genera mejores resultados, empleados satisfechos, motivados, clientes felices y un aumento de ingresos al acelerar los ciclos de venta, haciendo que los acuerdos con el cliente se cierren con mayor rapidez. Todo esto permite que los empleados encargados de la fabricación de los pedidos cometan menos errores gracias a que podrán visualizarlos con su respectivo estado, el cual puede ser: para fabricar, en camino y entregado.

Teniendo en cuenta lo mencionado anteriormente, el objetivo de la investigación es desarrollar una aplicación multiplataforma para el control de registros de pedidos en las microempresas de Cali basándose en la fábrica de pintura INDUBER.

\section{MARCO REFERENCIAL}

Las PYMES son microempresas que en la mayoría de los casos son familiares o con poca cantidad de 
empleados, que crean empresa para lograr ingresar a un mercado y permitir que la economía de la región se amplíe.

Ramírez, Bustamante \& Taborda (2012) realizan una aplicación denominada "Software prototipo de sistematización de pedidos e inventario de insumos para la elaboración de la comida rápida en la Empresa Coyote Good Food", aplicación que se realiza para comidas rápidas y sus pedidos. a la hora de hacer la implementación del sistema, se deben tener en cuenta varios factores, tales como: la forma en cómo se ejecuta el pedido para no generar dificultad al momento de usarlo, la cantidad de pedidos que puede visualizar el cocinero y las características de los productos para hacer el módulo de inventario. Esto conlleva a que la empresa empiece con un posicionamiento de marca antes sus clientes y mercado.

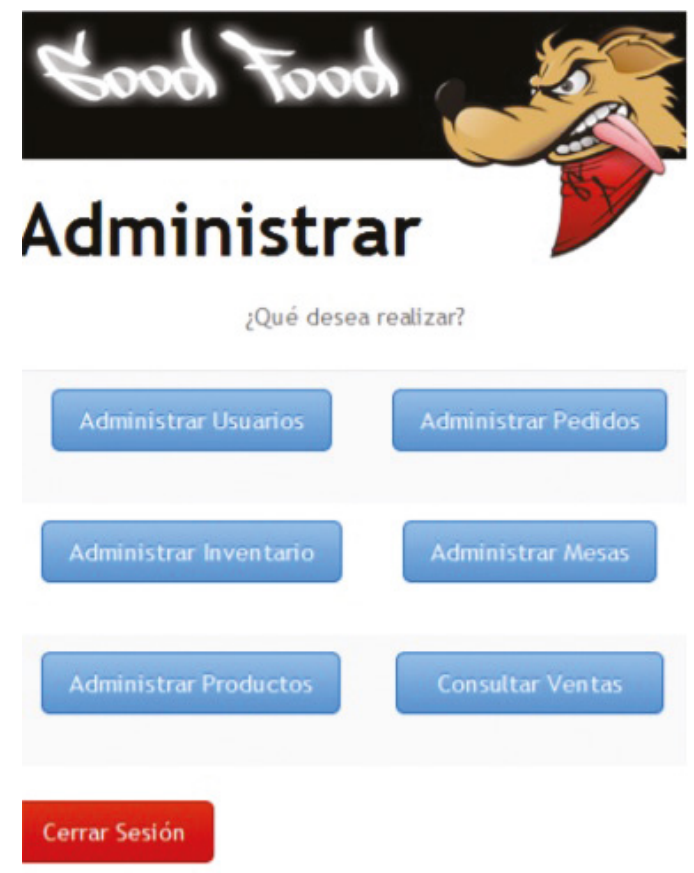

Figura 1. Pantalla de inicio del administrador para la aplicación Coyote Good Food. Fuente: Ramírez, Bustamante, \& Taborda (2012)

Así mismo, Cabello Fuentes (2013) desarrolló una aplicación denominada "Aplicación web de gestión de pedidos". En este proyecto se realizó una página web que integra a la página principal de la empre- sa, en la cual se permite hacer pedidos de los productos ofrecidos por dicha empresa, en caso de no haberse logrado hacer la integración completa con la página principal se ofrecía una plataforma en un servicio paralelo a la página. Este sistema permite no solo realizar pedidos por parte del cliente, sino que da permisos al empleado y al administrador para agregar pedidos que se hayan realizado por otros medios.

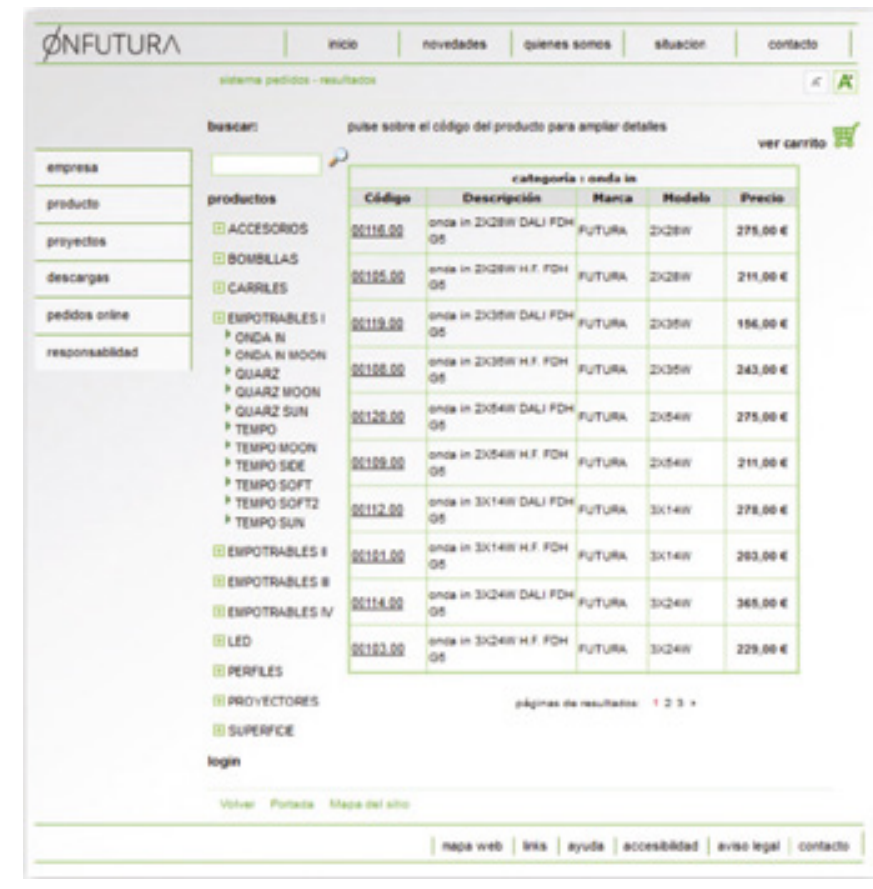

Figura 2. Pantalla producto de ONFUTURA.

(Fuentes, 2013)

\section{MARCO TEÓRICO}

Las PYMES, según plantea Seoane, E. (2005), son pequeñas y medianas empresas diseñadas para ofrecer productos o servicios de forma independiente en la mayoría de los casos, aunque se caracterizan por tener un número pequeño de empleados, ingresos y volumen de facturación, se identifican por ser el motor principal de empleo y riqueza dentro de un país por ser el mayor número de empresas constituidas dentro de este (Azucena, 2018). 
Hoy en día, el mundo permanece en un constante cambio, lo que obliga a todas las empresas a ir de la mano de estos para no perderse de las transformaciones actuales y quedar atrapadas en lo tradicional, ocasionando que desaparezcan con el paso del tiempo. Algo fundamental a tener en cuenta es la capacidad que tienen las Pymes para el aprovechamiento de estas tecnologías y el uso adecuado de sus datos e información para la fidelización de clientes, punto que siempre se tiene presente a la hora de proyectar una microempresa (Ravenna \& González, 2004).

Esto lleva a las pymes a enfrentar las tendencias digitales que van llegando día con día, adaptando los procesos que se manejan de forma tradicional e ir incursionando en la automatización de estos. "Datos de la Comisión Europea indican que las pequeñas y medianas empresas que adoptan nuevas tecnologías tienen una tasa de crecimiento de un $15 \%$ más alta, y 22\% más de ingresos que las pymes que no lo hacen" (Ortiz, 2017). Las nuevas tecnologías deben ser vistas por las pymes como una oportunidad para la obtención de clientes nuevos y, por lo tanto, el crecimiento de la producción, esto impacta de forma positiva la economía de la empresa y del país, considerando que las pymes abarcan el mayor número de empresas constituidas.

Al adaptar las nuevas tecnologías y realizar la automatización de procesos, mejora considerablemente el tiempo de producción y la eficiencia de los empleados (Fonseca Pinto, 2013), ya que disminuye tareas repetitivas y sin valor, lo que permite centrarse en actividades que requieran un mayor esfuerzo y dedicación, disminuye riesgos de cometer errores en el momento de toma y entrega de productos o servicios, gracias a la fiabilidad de los sistemas. Todo esto conlleva a una mejora en la calidad del servicio ofrecido por la empresa, ya que si no se cometen errores y se priorizan las tareas importantes, se obtienen resultados esperados en un menor tiempo (Sánchez, 2004).

Pero, ¿por qué es necesario automatizar los procesos en las pymes? La automatización es necesa- ria en cualquier nivel sin importar el tamaño de la empresa, pues es un hecho de que las nuevas tecnologías abarcan todos los ámbitos de nuestra vida, tanto personal como profesional (Ruiz, 2018). Actualmente en Colombia se está empezando a ver como una obligación automatizar algunos procesos, por ejemplo, la facturación electrónica que se ve amparada por el Decreto 2242 de 2015 (Morantes Manzano\& Carvajal Estupiñán, 2019), en el cual se establecen las condiciones de expedición de la factura electrónica con el fin de tener un mejor control fiscal.

Las pequeñas y medianas empresas deben aprender a aprovechar la tendencia del internet, ya que les permite exponerse ante un mercado mundial al cual no tenía acceso con anterioridad (Expansión, 2018).

Las empresas, sin importar su tamaño, deben acoplarse a estos cambios siguiendo las fases para su implementación de acuerdo con las TICs. Estas fases son:

1. Definición de necesidades y objetivos: consiste en la identificación de los objetivos empresariales de acuerdo con lo que necesita y para lo que se necesita.

2. Asesoramiento y gestión del proyecto: consiste en buscar la empresa que se encargará de la evaluación del sistema que necesita la empresa y el asesoramiento sobre la implementación de este.

3. Implantación y formación del personal: consiste en capacitar a los empleados sobre las herramientas que se implementarán en la empresa y al mismo tiempo darle inicio a su funcionamiento (Dicode, 2018).

\section{METODOLOGÍA Y RESULTADOS}

La fuente principal que nos suministrará la información es la fábrica de pinturas INDUBER, dado 
que esta empresa es la base para la realización de este proyecto y a la cual se le hará entrega de la aplicación desarrollada para su ejecución.

La información necesaria para el desarrollo de este proyecto se obtiene a través de una encuesta semiestructurada, creando una guía con los temas a cubrir, términos a usar y orden de las preguntas, la cual se realiza al administrador de la fábrica de pintura INDUBER, como se observa en la Tabla 1, y a sus clientes como lo indica en la Figura 3.

Tabla 1. Encuesta realizada al administrador de la fábrica de pinturas INDUBER.

\begin{tabular}{ll}
\hline \multicolumn{1}{c}{ PREGUNTA } & \multicolumn{1}{c}{ RESPUESTA } \\
\hline Nombre completo & $\begin{array}{l}\text { Bernardo A } \\
\text { Vásquez }\end{array}$ \\
\hline ¿Dónde se encuentra ubicado el negocio? & $\begin{array}{l}\text { Transversal 29 } \\
\text { \# 17 bis 124 }\end{array}$ \\
\hline $\begin{array}{l}\text { ¿Por qué medio realizan los pedidos sus } \\
\text { clientes normalmente? }\end{array}$ & Llamada \\
\hline $\begin{array}{l}\text { ¿Qué proceso realiza al momento de re- } \\
\text { gistrar un pedido hecho por un cliente? }\end{array}$ & $\begin{array}{l}\text { Tomar el } \\
\text { pedido en un } \\
\text { cuaderno }\end{array}$ \\
\hline $\begin{array}{l}\text { ¿Cuenta con clientes solamente dentro } \\
\text { de la ciudad de Cali? }\end{array}$ & No \\
\hline $\begin{array}{l}\text { ¿Cree usted que llevar el registro de pe- } \\
\text { didos de forma manual es adecuado? }\end{array}$ & No \\
\hline
\end{tabular}

¿Ha tenido algún contratiempo con el control de los pedidos de sus clientes? Es decir, ¿Ha olvidado hacer entregas en la No fecha y hora propuesta por el cliente?

¿Lleva un control adecuado sobre los productos que tiene en mostrador y los No insumos?

\begin{tabular}{ll}
\hline $\begin{array}{l}\text { ¿Considera importante o necesario } \\
\text { tener un historial de los pedidos que ha } \\
\text { entregado? }\end{array}$ & $\mathrm{Si}$ \\
\hline $\begin{array}{l}\text { ¿Cómo visualiza el proceso de registro } \\
\text { de pedidos dentro de su empresa en la } \\
\text { actualidad? }\end{array}$ & $\begin{array}{l}\text { Realizar el } \\
\text { proceso de } \\
\text { forma digital }\end{array}$ \\
\hline $\begin{array}{l}\text { ¿Cómo es la forma de pago que utiliza } \\
\text { dentro de la empresa para la venta de } \\
\text { productos? }\end{array}$ & Efectivo \\
\hline $\begin{array}{l}\text { ¿Implementaría el pago con tarjeta para } \\
\text { los clientes? }\end{array}$ & Sí \\
\hline
\end{tabular}

\begin{tabular}{ll}
\hline \multicolumn{1}{c}{ PREGUNTA } & RESPUESTA \\
\hline $\begin{array}{l}\text { ¿Considera necesario conocer el mo- } \\
\text { vimiento de sus clientes y productos } \\
\text { durante el mes? }\end{array}$ & Sí \\
\hline
\end{tabular}

Fuente. Elaboración propia

Las preguntas realizadas en las encuestas permiten hacer una caracterización de los clientes y ver la viabilidad de la implementación y uso posterior de la aplicación. Se analizan los datos de acuerdo con la información suministrada, dicha información se utiliza para dar inicio la fase de desarrollo de la aplicación y recolección de requerimientos.

En la Figura 3 se puede determinar que los clientes usan con frecuencia su dispositivo móvil, siendo esto apto para el uso de aplicaciones como la que se realiza.

\section{¿Con qué frecuencia utiliza el dispositvo móvil?}

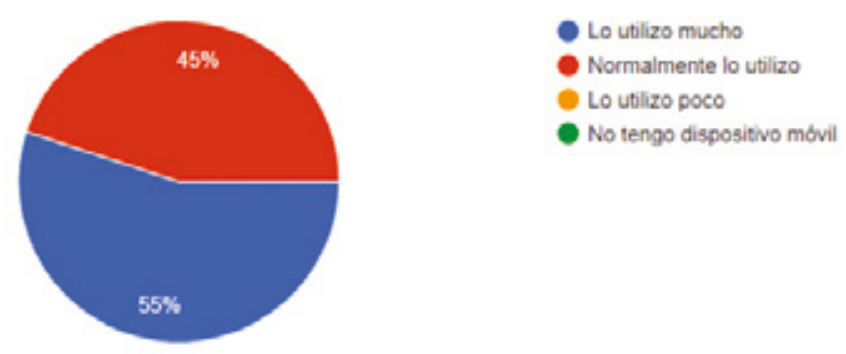

Figura 3. Frecuencia en que los clientes de la fábrica utilizan el dispositivo móvil.Fuente: Elaboración propia

La pregunta de la Figura 4 es de gran importancia para el desarrollo de la investigación, debido a que el realizar compras por internet puede permitir que la aplicación que se desarrollará sea fácilmente aceptada por los clientes. 


\section{¿Ha realizado o realiza compras por internet?}

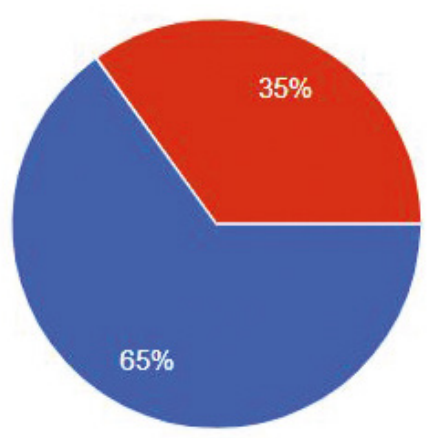

Figura 4. Indica si los clientes de la fabrica han realizado compras por internet. Fuente: Elaboración propia

En la Figura 5 se puede observar que los clientes de la empresa son fieles a ella por varios años, dicha información permite ser utilizada para campañas de fidelización con ellos.

\section{DESARROLLO DE LA APLICACIÓN}

Para este proyecto se escogió ICONIX, ya que esta es una metodología pesada-ligera que se creó a partir de RUP (Rational Unified Process) y XP (eXtreme Programming) para simplificar los procesos del desarrollo de software, sin perder el control sobre el ciclo de vida del proyecto y su documentación. ICONIX, al contrario de otras metodologías como RUP, no cuenta con un trabajo exigente para la documentación, ya que este solo deja lo necesario haciendo un uso diverso de UML (Unified Modeling Language), por lo que no es obligatorio hacer uso de todos sus diagramas.

\section{¿Hace cuánto tiempo es cliente de la fabrica INDUBER?}

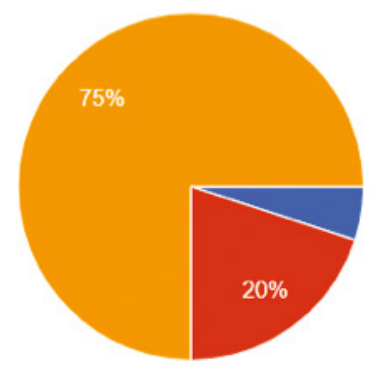

Menos de un año

De 1 a 3 años

Mas de 3 años

Figura 5. Tiempo de permanencia de los clientes de la fábrica. Fuente: Elaboración propia

Las fases que se van a seguir en esta metodología para mantener el control sobre las actividades son:

1. Revisión de los requisitos/ Análisis de Requisitos: se analizan los requisitos del sistema para construir el diagrama de clases. En esta fase se utilizan tres herramientas: requerimientos funcionales, modelo de dominio, modelo de casos de uso y modelo de interfaz de usuario.

- Requerimientos funcionales: Estos se diseñaron con base en las respuestas obtenidas en las encuestas realizadas al administrador y a 20 clientes de la fábrica de pinturas INDUBER. De estas encuestas se obtuvieron los requerimientos funcionales y se adecuan en los respectivos formatos como se indica en la Tabla 2. 
Tabla 2. Requerimiento funcional Iniciar Sesión.

\begin{tabular}{|c|c|}
\hline $\begin{array}{l}\text { IDENTIFICADOR: } \\
\text { R1 } \\
\end{array}$ & $\begin{array}{c}\text { NOMBRE: } \\
\text { Iniciar sesión. }\end{array}$ \\
\hline Tipo: & $\begin{array}{l}\text { REQUERIMIENTO QUE LO UTILIZA O } \\
\text { ESPECIALIZA: }\end{array}$ \\
\hline (NECESARIO/DESEABLE) & $\mathrm{R} 2$ \\
\hline Necesario & R5 \\
\hline $\begin{array}{l}\text { PRIORIDAD DE DESARROLLO: } \\
\text { Alta }\end{array}$ & DOCUMENTOS DE VISUALIZACIÓN ASOCIADOS: \\
\hline $\begin{array}{l}\text { ENTRADA: } \\
\text { • Correo } \\
\text { • Contraseña }\end{array}$ & $\begin{array}{l}\text { SALIDA: } \\
\text { Inicio de sesión exitoso }\end{array}$ \\
\hline
\end{tabular}

DESCRIPCIÓN:

Precondición: Ingresar datos solicitados por el sistema para el inicio de sesión.

Descripción: Una vez ingresados los datos se hará su validación en el sistema.

Postcondición: Se ingresará a la aplicación.

\section{MANEJO DE SITUACIONES ANORMALES:}

1. Los datos ingresados en el sistema son incorrectos, se muestra un mensaje de advertencia.

2. Dejar campos vacíos no habilitará el botón de inicio de sesión.

\section{CRITERIOS DE ACEPTACIÓN:}

1. El ingreso del usuario es validado por el sistema después de comprobar que los datos son correctos.

Fuente: Elaboración propia

- Modelo de dominio: Una vez realizados los requerimientos funcionales, estos se tomaron de base para realizar un modelado conceptual del problema a resolver con las clases y atributos a utilizar para el desarrollo de la aplicación.

- Modelo de casos de uso: Se realizó con base a los requerimientos funcionales y modelo de dominio, con esta herramienta se diagramó las tareas que tienen el administrador, los clientes y los empleados con el sistema.

- Modelo de interfaz de usuario: Se tuvo en cuenta el modelo de dominio para realizar el diseño de la interfaz de cada módulo que compone la aplicación.

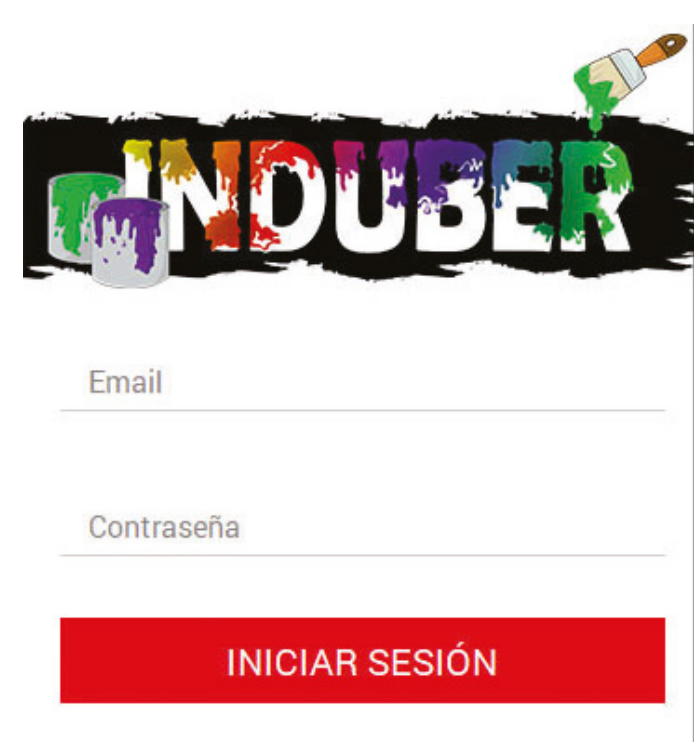

Figura 6. Interfaz de inicio de sesión. Fuente: Elaboración propia 


\begin{tabular}{|c|c|}
\hline Pedidos & Nuevo \\
\hline EN FABRICA & ENTREGADO \\
\hline \multicolumn{2}{|l|}{ Diana } \\
\hline \multicolumn{2}{|l|}{ Vinilo T1 Sanbercril } \\
\hline \multicolumn{2}{|l|}{ Michel } \\
\hline \multicolumn{2}{|l|}{ Vinilo T3 Sanbertex } \\
\hline \multicolumn{2}{|l|}{ Sandra } \\
\hline & 2019-05-28 5:15pm \\
\hline \multicolumn{2}{|l|}{ Vinil 2 Semilavable } \\
\hline \multicolumn{2}{|l|}{ Cesar } \\
\hline & 2019-05-27 12:02pm \\
\hline \multicolumn{2}{|l|}{ Estuco Pulimento Exterior } \\
\hline \multicolumn{2}{|l|}{ Dario } \\
\hline & 2019-05-27 9:20am \\
\hline \multicolumn{2}{|l|}{ Vinil 1 Lavable } \\
\hline \multicolumn{2}{|l|}{ Freddy } \\
\hline Vinilo T2 Sanbersol & 2019-05-27 8:00am \\
\hline & \\
\hline
\end{tabular}

Figura 7. Interfaz de Empleados de la fábrica. Fuente: Elaboración propia

2. Revisión del diseño preliminar /Análisis y Diseño Preliminar: En esta fase se realiza el diseño de las fichas de caso de uso por cada caso de uso. Las herramientas que se utilizan en esta fase son: casos de uso extendidos, diagrama de robustez y actualización de modelo de dominio.

- Casos de uso extendido: Se realizaron teniendo en cuenta los requerimientos funcionales y el diagrama de casos de uso para así obtener detalladamente las acciones del usuario con el sistema, tareas que deben realizar antes y después de cada interacción y así mismo conocer las posibles alteraciones del sistema en caso de no seguir el proceso adecuado para realizar una actividad.
- Diagrama de robustez: Se diseñó este diagrama en base a los casos de uso expandidos mostrando paso a paso las interacciones que tiene el usuario con el aplicativo móvil en cada uno de estos casos de uso.

- Actualización de modelo de dominio: Se realizaron ajustes al modelo de domino diseñado anteriormente, se adicionó la clase de devoluciones con sus respectivos atributos.

3. Revisión crítica del diseño/Diseño: En esta fase se reconocen todos los elementos que forman parte de nuestro sistema, para esto se hace uso de los diagramas de secuencia y el diagrama de dominio.

- Diagramas de secuencia: Se diseñaron con base en los diagramas de robustez para mostrar las interacciones entre cada objeto del sistema, los tiempos entre cada interacción y las respuestas obtenidas por cada una de estas acciones.

- Diagrama de dominio: Diseño final del modelado de dominio donde se muestra la relación y la solución para la automatización de la gestión de pedidos de la empresa.

4. Implementación: En esta fase se desarrollará el software que será entregado. Si es necesario se debe hacer uso del diagrama de componentes para apoyar el desarrollo.

\section{RESULTADOS}

Una vez diseñados los requerimientos funcionales, los casos de uso y los diagramas se dio paso a la codificación del aplicativo móvil. Para iniciar el desarrollo de este aplicativo se dividió el sistema en cuatro módulos: pedidos, consultas y reportes, usuarios e inventario. Cada módulo se caracteriza por:

- Pedidos: Este módulo se encarga de la visualización de los estados en que se encuentran cada pedido, esos estados son: En fábrica, En camino 
y Entregado. Dentro de cada estado se puede visualizar los datos del comprador, la fecha en que se tomó el pedido, la fecha a entregar el pedido y el pedido a realizar. También desde este módulo se puede realizar o crear un pedido escogiendo entre la variedad de productos de la empresa, siguiendo así el proceso de toma de pedidos.
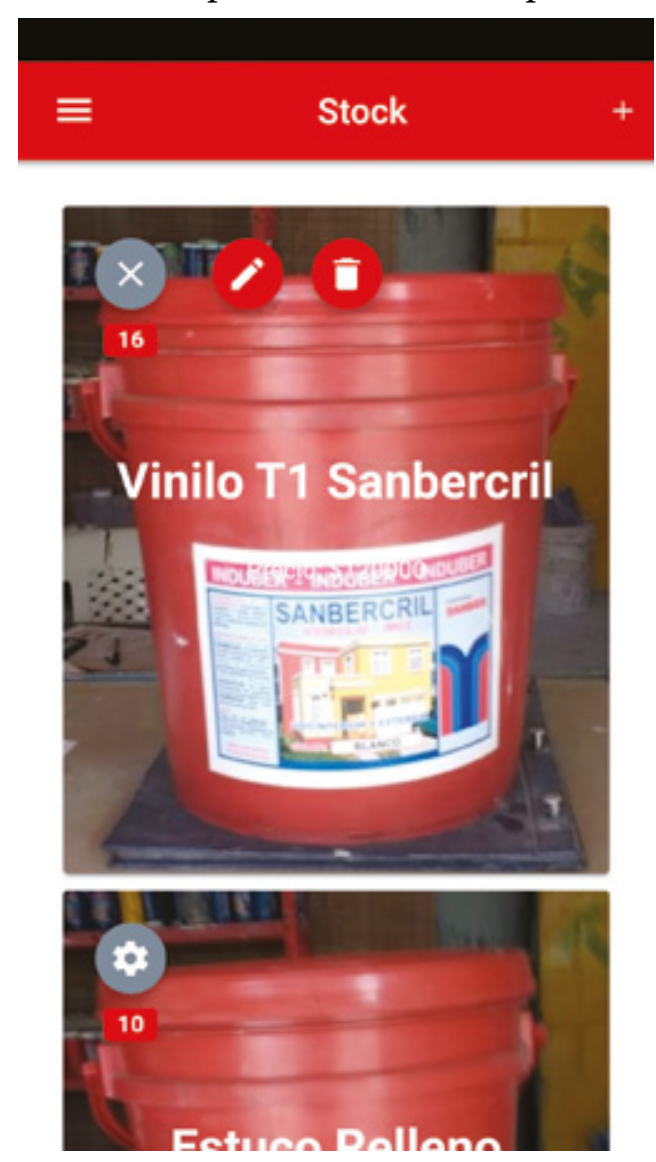

Figura 8. Pantalla de Inventario Fuente: Elaboración propia

- Consultas y Reportes: Este módulo se encarga de mostrarle al administrador el producto más vendido y los clientes más frecuentes; y permite generar reportes en PDF.

- Usuarios: Este módulo es el encargado de toda la gestión de las cuentas para los administradores, empleados y clientes donde cada cuenta tendrá un rol y privilegios o permisos diferentes.

- Inventario o Stock: Este módulo es el encargado de llevar o almacenar todos los productos de la empresa, con sus respectivos precios unitarios y cantidades existentes de cada producto.

\section{CONCLUSIONES}

Con este proyecto se puede concluir que la prioridad es tratar de que esas pequeñas empresas denominadas PYMES entren en una nueva era tecnológica con la cual pueden ampliar sus horizontes, destinando una inversión a automatizar procesos y adaptarse a los nuevos cambios que ello trae para beneficio común. Esto puede darse a través de aplicaciones móviles como la desarrollada con la empresa INDUBER, permitiendo así que el cliente se sienta parte fundamental de su modelo de negocio y logrando también a una fidelización de la marca.

Con el desarrollo de este proyecto se permitió cumplir con los objetivos planteados enfocados en el proceso de la gestión de pedidos. Esto, basado en la metodología ICONIX que al ser una metodología hibrida permite una mayor flexibilidad, la cual permitió un paso a paso del desarrollo del aplicativo de una manera más sencilla gracias a las herramientas y artefactos que ofrece, cabe mencionar que el uso de esta metodología permite un constante aprendizaje para su implementación.

Inicialmente el cliente no tenía presente la funcionalidad de devoluciones, pero una vez implementada la metodología se puede ver la flexibilidad que brinda ICONIX al momento de realizar cambios durante su proceso, es bastante favorable para simplificar el trabajo.

Muy importante resaltar la funcionalidad de reportes y consultas para una próxima actualización del sistema. Este módulo cuenta con un listado de los productos más vendidos y los clientes más frecuentes, sin embargo, puede plantearse como un trabajo futuro la adición de funciones más específicas como gráficas estadísticas, ingresos mensuales, anuales y semanales, de forma tanto individual como general e inversiones realizadas en los insumos de la empresa. 


\section{REFERENCIAS BIBLIOGRÁFICAS}

Azucena, D. (21 de agosto de 2018). PyMEs: ¿Qué son y cuál es su importancia? Obtenido de Emprendedores y negocios emprendedores y negocios aprende como hacer crecer tu negocio: https://emprendedoresynegocios.com/pymes-que-son/

Cámara de comercio Cali. (2 de agosto de 2018). Obtenido de Nueva iniciativa para acompañar el crecimiento de las microempresas: https://www.ccc. org.co/nueva-iniciativa-acompanar-crecimiento-las-microempresas/

Dicode. (17 de enero de 2018). Las pymes, ante el reto de las nuevas tecnologías. Obtenido de http://dicode. com/las-pymes-ante-el-reto-de-las-nuevas-tecnologias/

Expansión. (3 de octubre de 2018). Obtenido de La necesidad de implementar tecnología en una pyme: https:/expansion.mx/opinion/2018/10/03/ opinion-la-necesidad-de-implementar-tecnologia-en-una-pyme

Fonseca Pinto, D. E. (2013). Desarrollo e implementación de las TICS en las PYMES de Boyacá-Colombia.

Cabello Fuentes, J.L. y Patricio Guisado, M.A. (2013). Desarrollo de una aplicación web de gestión de pedidos. (Tesis de ingeniería técnica en informática de gestió). Universidad Carlos tercero de Madrid. Obtenido de https://e-archivo.uc3m.es/handle/10016/22018\#preview

Morantes Manzano, S., \& Carvajal Estupiñán, M. V. (2019). Implementación de la factura electrónica en Colombia a partir del decreto 2242 de 2015, en las MiPymes del barrio la merced de la ciudad de Cúcuta. (Tesis de grado especialización en tributaria). Universidad Libre seccional Cúcuta. Recuperado de: https://repository.unilibre.edu.co/handle/10901/15441
Ortíz, D. (11 de Octubre de 2017). Crecimiento de las pymes desde la automatización. Obtenido de https:// www.americaeconomia.com/analisis-opinion/crecimiento-de-las-pymes-desde-la-automatizacion

Ramírez, C. A., Bustamante, J. P., \& Taborda, L. Y. (2012). Software prototipo de sistematización de pedidos e inventario (tesis ingeniería de sistemas). Universidad Tecnológica de Pereira. Recuperado de: http://recursosbiblioteca.utp.edu.co/tesisd/textoyanexos/0053B562.pdf

Ravenna, M. C., \& González, M. L. (2004). Las tecnologías de la información en las pequeñas y medianas empresas mexicanas. Scripta Nova: revista electrónica de geografía y ciencias sociales, 8 , (21).

Ruiz, L. F. (17 de enero de 2018). Factura electrónica Colombia: ¿Qué se establece legalmente? Obtenido de https://www.siigo.com/blog/empresario/factura-electronica-colombia-que-se-establece-legalmente/

Sánchez, J. I. L. (2004). ¿Pueden las tecnologías de la información mejorar la productividad?. Universia Business Review, (1), 82-95.

Seoane, E. (2005). Estrategia para la implantación de nuevas tecnologías en Pymes. España: Ideaspropias Editorial SL.

\section{LOS AUTORES}

Diana Marcela Vásquez Pérez: Estudiante Ingeniería de Sistemas de la UNIAJC. Correo electrónico:vmarce05@gmail.com

Michel Chilito Sandoval: Estudiante Ingeniería de Sistemas de la UNIAJC. Correo electrónico: michelchilito@gmail.com

Tania Isadora Mora Pedreros: Ingeniera de Sistemas y Magister en Tecnología Educativa y Competencias Digitales. Docente Tiempo Completo UNIAJC. Correo electrónico: timora@admon.uniajc.edu.co 